\title{
Bilateral Erector Spinae Plane Block in a woman affected by chronic dorsal pain: a case report
}

\author{
P. Fusco1, S. Di Carlo2, F. Di Sanctis3, G. Degan2, A. Luciani2, P. Scimia4, F. \\ Marinangeli3. \\ 1San Salvatore Academic Hospital of L'Aquila, Department of Anesthesia and Intensive Care Unit, L'Aquila, \\ Italy. \\ 2University of Chieti, Department of Anesthesia- Resuscitation- Intensive and Pain Care, L'Aquila, Italy. \\ 3University of L'Aquila, Department of Life- Health and Environmental Sciences, L'Aquila, Italy. \\ 4A.S.S.T. of Cremona, Department of Anesthesia and Perioperative Medicine, Cremona, Italy.
}

Background and Aims:

Paravertebral block (PVB) is currently the gold standard for management of chronic thoracic pain. The Erector Spinae Plane Block (ESPB) could provide an adequate coverage of the chest wall as well as an effective analgesia in thoracic neuropathic pain.

Methods:

A 55 year-old woman with severe chronic dorsal pain for 2 years with referred NRS score 6 at rest and 9 for incident pain. She reported bilateral dorsal pain in from D3 to D7 level between the apex of left and right scapula. She was on treatment with transdermal fentanyl $50 \mathrm{mcg} / \mathrm{h}$, oral morphine 8 drops as needed, paracetamol $1000 \mathrm{mg}$ each 8 hours. Radiological examinations excluded spinal pathologies.

Results

For the first 15 days, the patient reported a complete benefit with NRS score 1. Subsequently the pain recurred, but with much lower intensity. Three months after the procedure, NRS score was 4-5, and the patient was on treatment with transdermal fentanyl $25 \mathrm{mcg} / \mathrm{h}$ and paracetamol.

Conclusions:

ESPB could provide a long-lasting analgesia in chronic dorsal pain, allowing to reach many levels of dermatomes. The interruption of the painful stimulus probably provided a remodulation of pain, wich recurred with a lower intensity. 